\title{
Protective effect of bilobalide on learning and memory impairment in rats with vascular dementia
}

\author{
WEI-ZU LI ${ }^{1,2^{*}}$, WANG-YANG WU ${ }^{1,2^{*}}$, HUAN HUANG ${ }^{1,2}$, YANG-YANG WU ${ }^{1,2}$ and YAN-YAN YIN ${ }^{1,2}$ \\ ${ }^{1}$ Department of Pharmacology, Key Laboratory of Anti-Inflammatory and Immunopharmacology, Ministry of Education; \\ ${ }^{2}$ Key Laboratory of Chinese Medicine Research and Development, State Administration of Traditional Chinese Medicine, \\ Anhui Medical University, Hefei, Anhui 230032, P.R. China
}

Received February 2, 2013; Accepted June 24, 2013

DOI: $10.3892 / \mathrm{mmr} .2013 .1573$

\begin{abstract}
Vascular dementia (VD) is the second most common type of dementia in the elderly. Currently, there are no effective drugs for preventing or decreasing the progression of dementia. Bilobalide (BB) is a monomer extracted from Ginkgo biloba leaves. The present study investigated the neuroprotective effects of BB in a two-vessel occlusion (2-VO)-induced $\mathrm{VD}$ rat model. The results showed that $\mathrm{BB}(4$ and $8 \mathrm{mg} / \mathrm{kg})$ significantly protected VD rats against cognitive deficits in the Morris water maze. Biochemical assessment showed that BB (4 and $8 \mathrm{mg} / \mathrm{kg}$ ) increased superoxide dismutase (SOD) activity and glutathione (GSH) content, and decreased nitric oxide synthase (NOS) activity and malondialdehyde (MDA) content. Additionally, BB (4 and $8 \mathrm{mg} / \mathrm{kg}$ ) was found to alleviate neuronal apoptosis and to reduce the expression of tumor necrosis factor- $\alpha$ (TNF- $\alpha$ ) in the brain cortex and the hippocampal CA1 region in VD rats. These results suggest that BB provides protection against learning and memory impairment by reducing free radical injury and inhibiting neuronal apoptosis in the brain cortex and hippocampal CA1 region in VD rats.
\end{abstract}

\section{Introduction}

Vascular dementia (VD) occurs as a result of ischemic injury or oligemia to brain areas, which leads to a progressive cognition decline, functional ability impairment and behavioral problems (1). VD is considered the second most common

Correspondence to: Professor Yan-Yan Yin, Department of Pharmacology, Key Laboratory of Anti-Inflammatory and Immunopharmacology, Ministry of Education, Anhui Medical University, No. 81 Meishan Road, Hefei, Anhui 230032, P.R. China

E-mail: yinyanyan5678@126.com

${ }^{*}$ Contributed equally

Key words: vascular dementia, Morris water maze, oxidative stress, tumor necrosis factor- $\alpha$ dementia and prevalence of VD is increased, accompanied by a corresponding increase in elderly population. Currently, there is no effective cure for VD, thus investigators have focused on preventing further brain damage.

The standardized Ginkgo biloba extract, EGb 761, has been reported to have neuroprotective effects against various neurological disorders, such as Alzheimer's disease, ischemia and depression (2-4). Although these results suggest the possibility of using the Ginkgo biloba extract as an anti-dementia drug, the properties of each constituent of the extract should be analyzed to develop an optimally effective anti-dementia drug. EGb 761 contains two groups of bioactive constituents, the flavonoids (24\%) and the terpenoids (6\%), which have been actively investigated for their neuroprotective and neuromodulatory activities (5). It has been reported that the effectiveness of the Ginkgo biloba extract EGb 761 in a middle cerebral artery occlusion (MCAO) model was exclusively due to the presence of bilobalide (BB), a sesquiterpene lactone that constitutes $2.9-3.2 \%$ of the extract $(6,7)$. Accumulating evidence suggest that BB has neuroprotective effects $(2,8,9)$. Optimum efficacy usually requires extracts at high doses $(50-200 \mathrm{mg} / \mathrm{kg})$, while using lower doses of BB may result in an equally high efficacy.

Chronic cerebral hypoperfusion is mimicked in animals by bilateral common carotid artery occlusion (2-vessel occlusion, 2-VO), resulting in the establishment of a suitable model for investigating the mechanisms of VD (10). The aim of the present study was to investigate the effects of $\mathrm{BB}$, a specific constituent of the Ginkgo biloba extract EGb 761, on VD rats. Consequently, we investigated the effects of $\mathrm{BB}$ on the learning and memory dysfunction in VD rats. BB was hypothesized to improve the learning and memory dysfunction in VD rats due to its antioxidant and antiapoptotic properties. Furthermore, we investigated the effects of BB on oxidative injury, neuronal apoptosis and the expression of tumor necrosis factor- $\alpha$ (TNF- $\alpha$ ) in VD rats.

\section{Materials and methods}

Drugs and reagents. Bilobalide (content of BB, >98\%; Fig. 1) was obtained from the Creative Pharmaceutical Technology Co., Ltd. (Hefei, China). Streptavidin-biotin complex and diaminobenzidine (DAB) staining kits were purchased from ZSGB-BIO (Beijing, China). Antibody against TNF- $\alpha$ was 
purchased from Beijing Biosynthesis Biotechnology Co., Ltd. (China). Drugs were dissolved in distilled water, and all the additional chemicals were of the highest analytical grade available.

Animals and treatment. The rodent experiments were appropriately performed to minimize animal suffering and were in accordance with the animal protocols approved by the local authorities in Anhui, China. Adult male Sprague Dawley rats (2 to 3-month-old, 180-200 g) were obtained from the Center of Laboratory Animal of Anhui, China (Grade II, Certificate no. SCXK 2005-001). The rats were housed under conditions of controlled lighting (12-h light/dark cycle) and temperature $\left(25^{\circ} \mathrm{C}\right)$. All the animals were allowed ad libitum access to food and water, and were allowed to acclimatize for $\geq 3$ days prior to experimentation.

The rats were randomly divided into 7 groups: the sham-surgery, model, BB (2, 4 and $8 \mathrm{mg} / \mathrm{kg}$ ), Nimotop (Nimo, $10.5 \mathrm{mg} / \mathrm{kg}$ ) and Huperzine A (Hupa, $0.047 \mathrm{mg} / \mathrm{kg}$ ) groups. Nimo and Hupa, which are standard drugs for dementia treatment, were used as positive controls. The animals were anesthetized with $10 \%$ chloral hydrate $[3 \mathrm{ml} / \mathrm{kg}$, intraperitoneally (i.p.)]. Surgical operation was conducted as previously described (11). Briefly, through a midline cervical incision, the bilateral common carotid arteries of each rat were carefully separated from the cervical sympathetic and vagal nerves. The bilateral common carotid arteries were then exposed and ligated with 4-0 silk sutures in the permanent 2-VO group. The rats of the sham-operated group underwent the same surgical operation without carotid artery ligations. Mean body temperature (taken rectally) was maintained during surgery at $37.0 \pm 0.5^{\circ} \mathrm{C}$. The rats of the BB $(2,4$ and $8 \mathrm{mg} / \mathrm{kg})$, Nimo $(10.5 \mathrm{mg} / \mathrm{kg})$ and Hupa $(0.047 \mathrm{mg} / \mathrm{kg})$ groups were treated with the respective drugs [intragastric (i.g.) administration] for 60 days from day 1 following surgery. The rats of the sham-surgery group were administered equivalent volumes of vehicle.

Morris water maze test. The Morris water maze (MWM) consists of a black 1.6-m diameter circular pool. The pool was divided into 4 equal hypothetical quadrants and filled with water $\left(24^{\circ} \mathrm{C}\right.$; depth, $\left.30 \mathrm{~cm}\right)$. In the middle of a certain quadrant, there was a submerged escape platform $(10 \mathrm{~cm}$ in diameter), which was placed $2 \mathrm{~cm}$ below the surface of the water. The water maze apparatus, rat handling, and general testing procedure have been previously described (12).

All the rats were released into water (facing the wall) at 4 distinct starting quadrant points and were given 4 trials/day (90 sec/trial) from 56 to 59 days following surgery, for 4 consecutive days. Using an on-line image video tracking system, escape latency (sec) was recorded to determine changes in learning dysfunction. On day 60 , following completion of all the learning trials for all the rats, the platform was removed from the pool, and each rat received one 90 -sec swim probe trial. The time of swimming in the quadrant of platform (STP) and the number of rats crossing the platform (NCP) were recorded to determine changes in memory dysfunction. Behavioral data were analyzed using analysis of variance.

Detection of superoxide dismutase (SOD), nitric oxide synthase (NOS) activity, and malondialdehyde (MDA)

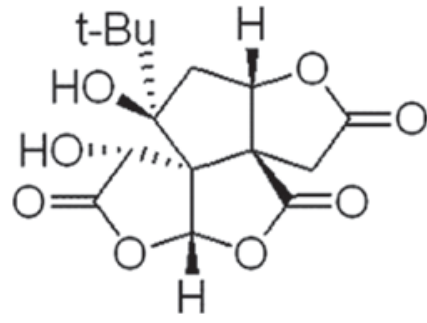

Bilobalide

Figure 1. Chemical structure of bilobalide (BB).

glutathione (GSH) content. Following MWM testing, 8 rats from each group were anesthetized and sacrificed by decapitation. The brain of each rat was dissociated and stored at $-80^{\circ} \mathrm{C}$ until assessment. Superoxide dismutase (SOD) and nitric oxide synthase (NOS) activity, as well as malondialdehyde (MDA) and glutathione (GSH) content were determined using the appropriative detection kits (Nianjing Jiancheng Bioengineering Institute, Nanjing, China).

Histological examination. Following MWM testing, 5 rats from each group were anesthetized with $10 \%$ chloral hydrate $(3.0 \mathrm{ml} / \mathrm{kg})$. Following animal sacrifice, the brain of each rat was removed, fixed in $4 \%$ paraformaldehyde and embedded in paraffin. The brain tissues were sliced $(5-\mu \mathrm{m})$ using a section cutter (Leica Biosystems, Wetzlar, Germany). The sections were then stained with hematoxylin and eosin (H\&E) and examined under a light microscope.

Hoechst 33258 staining. For nuclear staining, paraffin sections were dehydrated through a gradient of xylene and ethanol, and then rinsed with phosphate-buffered saline (PBS). The sections were incubated with $25 \mathrm{mM}$ Hoechst 33258 (ZSGB-BIO) for $15 \mathrm{~min}$ at $37^{\circ} \mathrm{C}$, washed with PBS (pH 7.2-7.5), mounted onto slides using antifade mounting medium, and then examined using fluorescence microscopy (Ex/Em, 352/461 nm) (Olympus Optical, Tokyo, Japan).

Immunohistochemistry. Paraffin sections were cut $(5-\mu \mathrm{m})$ and fixed to slides to ensure adhesion. The sections were incubated with hydrogen peroxide (3\%) to inactivate endogenous peroxidase, and non-immune goat serum was used as a blocking agent. The sections were incubated with the primary antibody against TNF- $\alpha$ (1:100) overnight at $4^{\circ} \mathrm{C}$. Immunostaining was visualized by the peroxidase method with a biotinylated anti-rabbit secondary antibody and DAB oxidation. The sections were resin-mounted and examined using a fluorescence microscope (Nikon, Japan). The immunoreactive neurons which were stained brown were observed, and images were captured under the microscope. Five sections/group and the three high-power fields of the hippocampal CA1 region and the brain cortex/section of the same magnification $(x 400)$ were used for quantitative analysis. The mean optical density of positive neurons in each section was measured using the Image-Pro Plus 6.0 analysis system to determine TNF- $\alpha$ expression.

Statistical analysis. Intergroup differences were analyzed using one-way ANOVA. The Student's t-test was used to 
Table I. Effect of BB on mean escape latencies in VD rats.

Mean escape latencies (sec)

\begin{tabular}{|c|c|c|c|c|c|}
\hline \multirow[b]{2}{*}{ Group } & \multirow[b]{2}{*}{ Dose $(\mathrm{mg} / \mathrm{kg})$} & \\
\hline & & Day 1 & Day 2 & Day 3 & Day 4 \\
\hline Sham & - & $58.99 \pm 37.20$ & $31.19 \pm 28.77$ & $19.82 \pm 15.03$ & $12.15 \pm 9.62$ \\
\hline Model & - & $80.11 \pm 22.96^{\mathrm{a}}$ & $64.27 \pm 34.37^{\mathrm{a}}$ & $56.48 \pm 36.67^{\mathrm{a}}$ & $55.61 \pm 35.40^{\mathrm{a}}$ \\
\hline Nimo & 10.5 & $68.03 \pm 34.96$ & $50.25 \pm 31.28$ & $43.82 \pm 31.15$ & $40.22 \pm 21.35^{b}$ \\
\hline Hupa & 0.047 & $56.33 \pm 32.08^{c}$ & $45.96 \pm 31.59^{b}$ & $30.36 \pm 27.81^{\mathrm{c}}$ & $26.24 \pm 26.75^{\mathrm{c}}$ \\
\hline \multirow[t]{3}{*}{$\mathrm{BB}$} & 2 & $72.35 \pm 25.05$ & $49.87 \pm 34.66$ & $33.58 \pm 30.82^{c}$ & $22.12 \pm 18.37^{c}$ \\
\hline & 4 & $77.47 \pm 24.35$ & $55.95 \pm 31.70$ & $29.90 \pm 24.65^{c}$ & $28.75 \pm 22.05^{\mathrm{c}}$ \\
\hline & 8 & $68.90 \pm 29.56$ & $52.99 \pm 33.45$ & $32.65 \pm 30.36^{c}$ & $27.23 \pm 25.14^{\mathrm{c}}$ \\
\hline
\end{tabular}

VD rats exhibited learning and memory impairment. BB treatment significantly reduced the mean escape latencies (particularly on days 3 and 4). Data are the mean $\pm \mathrm{SD}(\mathrm{n}=8)$. ${ }^{\mathrm{a}} \mathrm{P}<0.01 \mathrm{vs}$. sham-surgery group; ${ }^{\mathrm{b}} \mathrm{P}<0.05,{ }^{\mathrm{c}} \mathrm{P}<0.01$ vs. model group. $\mathrm{BB}$, bilobalide; VD, vascular dementia. Nimo, Nimotop-treated rats; Hupa, Huperzine A-treated rats.

determine significant differences between groups. Results were expressed as the mean $\pm \mathrm{SD}$. $\mathrm{P}<0.05$ was considered to indicate a statistically significant difference.

\section{Results}

Effects of $B B$ on the learning and memory ability of VD rats. MWM was used to determine the learning and memory ability of rats. In the memory training experiment, the mean escape latencies in the rats of all the groups were significantly reduced with increasing time. The mean escape latencies in the VD rats of the model group were significantly increased compared with the model group $(\mathrm{P}<0.01)$. The mean escape latencies were differently decreased following $\mathrm{BB}(2,4$ and $8 \mathrm{mg} / \mathrm{kg})$, Nimo and Hupa treatment $(\mathrm{P}<0.05$ or $<0.01)$ (Table I). In addition, NCP and STP in the rats of the model group were also significantly reduced compared with the sham-surgery group $(\mathrm{P}<0.05$ or $<0.01)$. NCP $(\mathrm{P}<0.05)$ and STP $(\mathrm{P}<0.05$ or $<0.01)$ in the rats of the $\mathrm{BB}(2,4$ and $8 \mathrm{mg} / \mathrm{kg})$, Nimo and Hupa groups were differentially decreased compared with the model group (Table II).

Effects of BB on neuronal morphology in the brain cortex and hippocampal CAl region. $\mathrm{H} \& \mathrm{E}$ and Hoechst 33258 staining were used to investigate the neuronal morphology and apoptosis of the brain cortex and the hippocampal CA1 region of the rats. $H \& E$ staining indicated that there were no significant neuronal abnormalities in the brain cortex and the hippocampal CA1 region of the rats in the sham-surgery group (Fig. 2A). However, the rats in the model group exhibited acidophilia degeneration, nuclear condensation and disorder of the array of neurons in these regions (Fig. 2B). Neuronal degeneration was alleviated in the rats of the BB (4 and $8 \mathrm{mg} / \mathrm{kg}$ ), Nimo and Hupa groups compared with the model group (Fig. 2).

We examined the neuronal apoptosis of the brain cortex and the hippocampal CA1 region of rats using Hoechst 33258 staining. The average number of apoptotic cells in these regions, which appear smaller than normal and in which the chromatin appears to be condensed, were counted $(n=5)$. The
Table II. Effect of BB on STP and NCP in VD rats.

\begin{tabular}{lccc}
\hline Group & Dose $(\mathrm{mg} / \mathrm{kg})$ & STP $(\mathrm{sec})$ & NCP \\
\hline Sham & - & $32.90 \pm 5.89$ & $6.38 \pm 2.62$ \\
Model & - & $25.56 \pm 5.34^{\mathrm{a}}$ & $1.50 \pm 1.60^{\mathrm{b}}$ \\
Nimo & 10.5 & $31.28 \pm 6.62$ & $4.63 \pm 2.97^{\mathrm{c}}$ \\
Hupa & 0.047 & $35.19 \pm 7.84^{\mathrm{c}}$ & $5.00 \pm 2.33^{\mathrm{d}}$ \\
BB & 2 & $28.44 \pm 5.77$ & $4.75 \pm 3.20^{\mathrm{c}}$ \\
& 4 & $33.83 \pm 9.00^{\mathrm{c}}$ & $4.63 \pm 3.54^{\mathrm{c}}$ \\
& 8 & $33.39 \pm 8.48^{\mathrm{c}}$ & $5.63 \pm 2.56^{\mathrm{d}}$ \\
\hline
\end{tabular}

Swimming time in the quadrant of platform (STP) and the average number of rats crossing the platform site (NCP) were significantly decreased in the rats of the model group. BB (4 and $8 \mathrm{mg} / \mathrm{kg}$ ) and Hupa treatment increased STP and NCP. Data are the mean \pm SD $(\mathrm{n}=8) .{ }^{\mathrm{a}} \mathrm{P}<0.05,{ }^{\mathrm{b}} \mathrm{P}<0.01$ vs. sham-surgery group; ${ }^{\mathrm{c}} \mathrm{P}<0.05,{ }^{\mathrm{d}} \mathrm{P}<0.01$ vs. model group. BB, bilobalide; VD, vascular dementia; Nimo, Nimotop-treated rats; Hupa, Huperzine A-treated rats.

percentage of nuclear condensation in the brain cortex and the hippocampal CA1 region in the rats of the model group was significantly increased compared with sham-surgery group $(\mathrm{P}<0.05$ or $<0.01)$. The percentage of nuclear condensation in the brain cortex and the hippocampal CA1 region in the rats of the BB (4 and $8 \mathrm{mg} / \mathrm{kg}$ ), Hupa and Nimo groups was differentially decreased compared with the model group $(\mathrm{P}<0.05$ or $<0.01$; Fig. 3).

Effects of $B B$ on brain tissue SOD, NOS activities and $M D A, G S H$ contents. SOD activity and GSH content were significantly decreased $(\mathrm{P}<0.01)$, while the MDA content and NOS activity were clearly increased $(\mathrm{P}<0.01)$ in the rats of the model group compared with the sham-surgery group. BB 2, 4 and $8 \mathrm{mg} / \mathrm{kg}$ ), Nimo and Hupa treatment differentially increased SOD activity and GSH content $(\mathrm{P}<0.05$ or $<0.01)$, but decreased MDA content and NOS activity in VD rats 

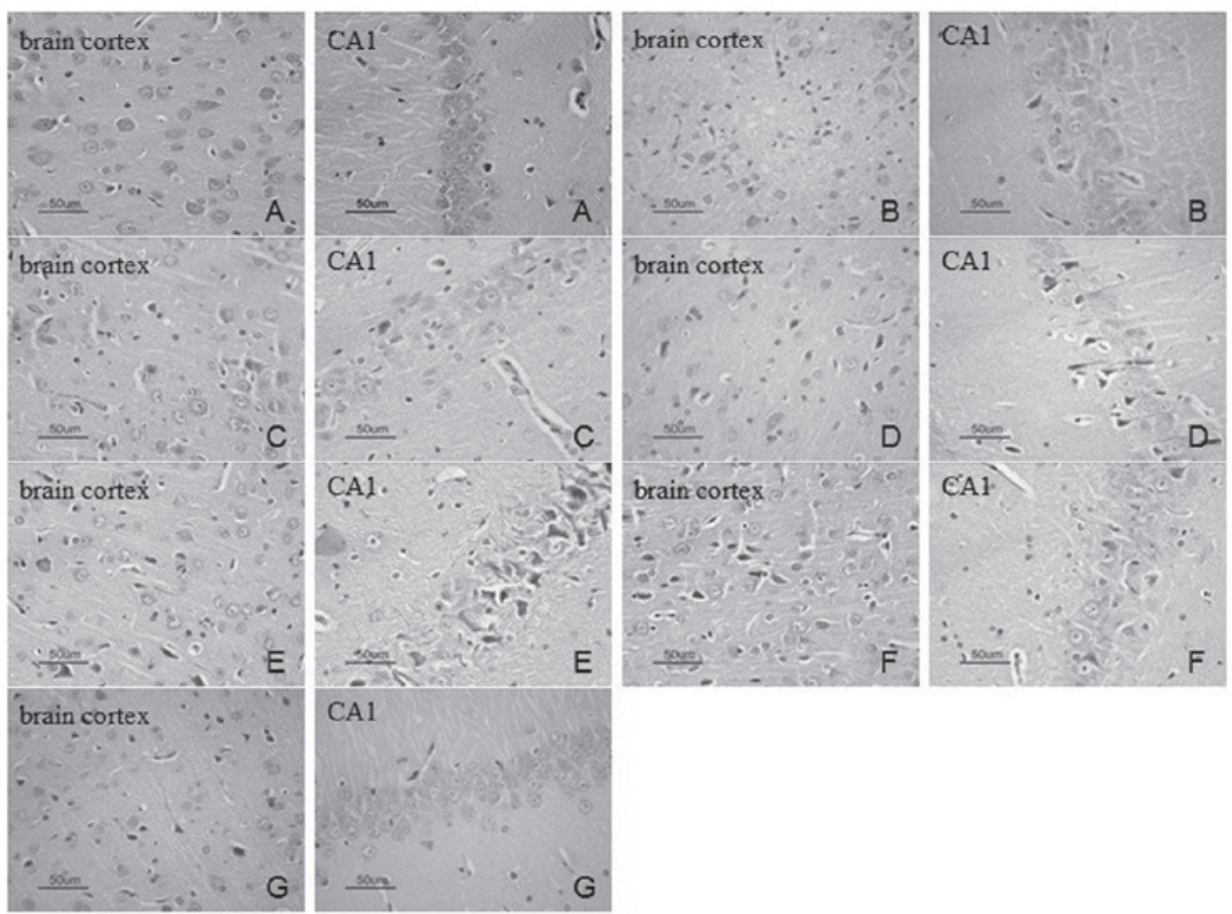

Figure 2. Effects of bilobalide (BB) on the neuronal morphology in the brain cortex and hippocampal CA1 region of rats (H\&E staining; magnification, $\mathrm{x} 400$ ). No significant neuronal abnormalities in the brain cortex and hippocampal CA1 region of the rats in the sham-surgery group were observed, while the rats in the model group exhibited degeneration of neurons and disorder of the array of neurons. In BB (4 and $8 \mathrm{mg} / \mathrm{kg})$, Nimo and Hupa groups, the number of acidophilia degeneration and nuclear condensation was decreased. (A Sham-surgery, (B) model, (C) Nimo, (D) Hupa, (E) BB 2 mg/kg, (F) BB 4 mg/kg and (G) BB $8 \mathrm{mg} / \mathrm{kg}$ groups ( $\mathrm{n}=5$ rats/group).

Table III. Effects of BB on brain tissue SOD activity and MDA content in VD rats.

\begin{tabular}{lccc}
\hline Group & $\begin{array}{c}\text { Dose } \\
(\mathrm{mg} / \mathrm{kg})\end{array}$ & $\begin{array}{c}\text { SOD } \\
(\text { KU/g protein })\end{array}$ & $\begin{array}{c}\text { MDA } \\
(\mu \mathrm{mol} / \mathrm{g} \text { protein })\end{array}$ \\
\hline Sham & - & $125.54 \pm 8.06$ & $3.31 \pm 0.78$ \\
Model & - & $104.78 \pm 10.63^{\mathrm{a}}$ & $4.91 \pm 0.62^{\mathrm{a}}$ \\
Nimo & 10.5 & $119.53 \pm 9.80^{\mathrm{b}}$ & $3.76 \pm 0.94^{\mathrm{b}}$ \\
Hupa & 0.047 & $117.67 \pm 10.85^{\mathrm{b}}$ & $3.87 \pm 0.90^{\mathrm{b}}$ \\
BB & 2 & $115.80 \pm 9.23^{\mathrm{b}}$ & $4.08 \pm 0.71^{\mathrm{b}}$ \\
& 4 & $116.88 \pm 8.96^{\mathrm{b}}$ & $3.83 \pm 0.84^{\mathrm{b}}$ \\
& 8 & $118.46 \pm 6.93^{\mathrm{c}}$ & $3.65 \pm 0.59^{\mathrm{c}}$ \\
\hline
\end{tabular}

Data are the mean $\pm \mathrm{SD}(\mathrm{n}=8)$. ${ }^{\mathrm{a}} \mathrm{P}<0.01$ vs. sham-surgery group; ${ }^{\mathrm{b}} \mathrm{P}<0.05,{ }^{\mathrm{c}} \mathrm{P}<0.01$ vs. model group. $\mathrm{BB}$, bilobalide; $\mathrm{SOD}$, superoxide dismutase; MDA, malondialdehyde; VD, vascular dementia; Nimo, Nimotop-treated rats; Hupa, Huperzine A-treated rats.

compared with the rats in the model group $(\mathrm{P}<0.05$ or $<0.01)$ (Tables III and IV).

Effect of $B B$ on the expression of TNF- $\alpha$ in the brain cortex and the hippocampal CAl region. The effect of BB on TNF- $\alpha$ immunoreactivity in the brain cortex and the hippocampal CA1 region in VD rats was investigated. Under normal conditions, immunoreactive cells are abundant and appear brown in color following immunohistochemistry. Many TNF- $\alpha$ immu-
Table IV. Effects of BB on brain tissue NOS activity and GSH content in VD rats.

\begin{tabular}{lccc}
\hline Group & $\begin{array}{c}\text { Dose } \\
(\mathrm{mg} / \mathrm{kg})\end{array}$ & $\begin{array}{c}\text { NOS } \\
(\text { KU/g protein })\end{array}$ & $\begin{array}{c}\text { GSH } \\
(\mu \mathrm{mol} / \mathrm{g} \text { protein })\end{array}$ \\
\hline Sham & - & $0.44 \pm 0.09$ & $29.06 \pm 6.50$ \\
Model & - & $0.75 \pm 0.15^{\mathrm{a}}$ & $16.30 \pm 4.29^{\mathrm{a}}$ \\
Nimo & 10.5 & $0.54 \pm 0.17^{\mathrm{b}}$ & $23.11 \pm 5.57^{\mathrm{b}}$ \\
Hupa & 0.047 & $0.53 \pm 0.16^{\mathrm{b}}$ & $23.45 \pm 5.28^{\mathrm{c}}$ \\
BB & 2 & $0.61 \pm 0.15$ & $22.16 \pm 5.96^{\mathrm{b}}$ \\
& 4 & $0.54 \pm 0.19^{\mathrm{b}}$ & $22.32 \pm 3.83^{\mathrm{b}}$ \\
& 8 & $0.51 \pm 0.17^{\mathrm{c}}$ & $24.57 \pm 3.60^{\mathrm{c}}$ \\
\hline
\end{tabular}

Data are the mean $\pm \mathrm{SD}(\mathrm{n}=8)$. ${ }^{\mathrm{a}} \mathrm{P}<0.01$ vs. sham-surgery group; ${ }^{\mathrm{b}} \mathrm{P}<0.05,{ }^{\mathrm{c}} \mathrm{P}<0.01$ vs. model group. $\mathrm{BB}$, bilobalide; NOS, nitric oxide synthase; GSH, glutathione; VD, vascular dementia; Nimo, Nimotop-treated rats; Hupa, Huperzine A-treated rats.

noreactive neuronal cells were observed in the brain cortex and the hippocampal CA1 region in the rats of the model group compared with the sham-surgery group. However, the number of TNF- $\alpha$ immunoreactive cells in the BB (4 and $8 \mathrm{mg} / \mathrm{kg}$ ), Nimo and Hupa-treated rats was significantly decreased compared with the model group (Fig. 4).

The mean optical density of the brain cortex and the hippocampal CA1 region in the rats of the model group was significantly increased compared with the sham-surgery group 

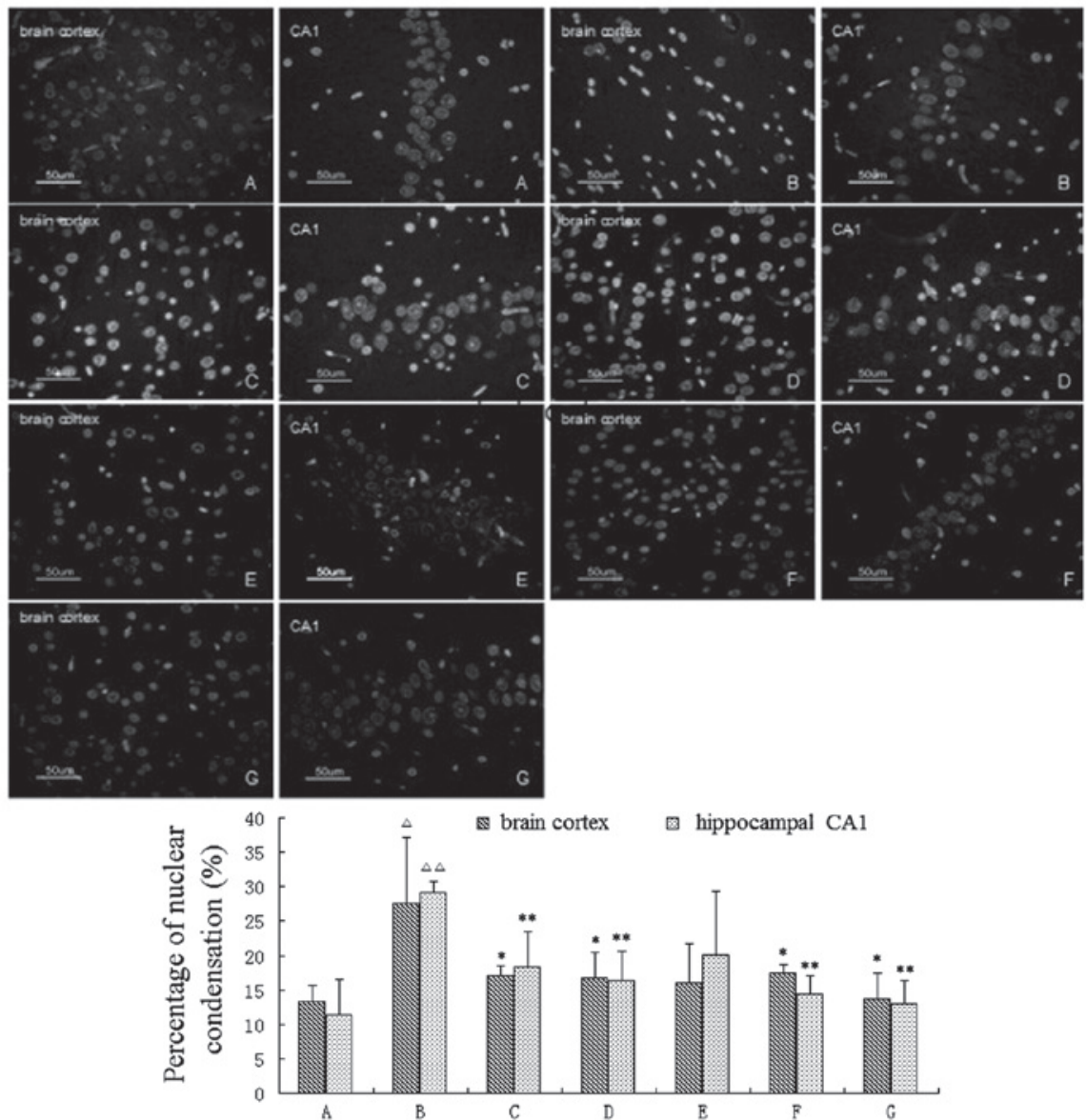

Figure 3. Effects of bilobalide (BB) on neuronal apoptosis in the brain cortex and hippocampal CA1 region (Hoechst 33258 staining; magnification, $\mathrm{x} 400$ ). Neuronal apoptosis in the brain cortex and hippocampal CA1 region was significantly increased in the rats of the (B) model group compared to the (A) sham-surgery group. However, the number of apoptotic cells in the brain cortex and the hippocampal CA1 region in the rats of the BB (4 and $8 \mathrm{mg} / \mathrm{kg}$ ), Nimo and Hupa groups were significantly decreased. (A) Sham-surgery, (B) model, (C) Nimo, (D) Hupa, (E) BB 2 mg/kg, (F) BB 4 mg/kg and (G) BB 8 mg/kg groups ( $\mathrm{n}=5$ rats/group). ${ }^{\triangle} \mathrm{P}<0.05,{ }^{\triangle} \mathrm{P}<0.01$ vs. sham-surgery group; ${ }^{\prime} \mathrm{P}<0.05,{ }^{* * *} \mathrm{P}<0.01$ vs. model group.
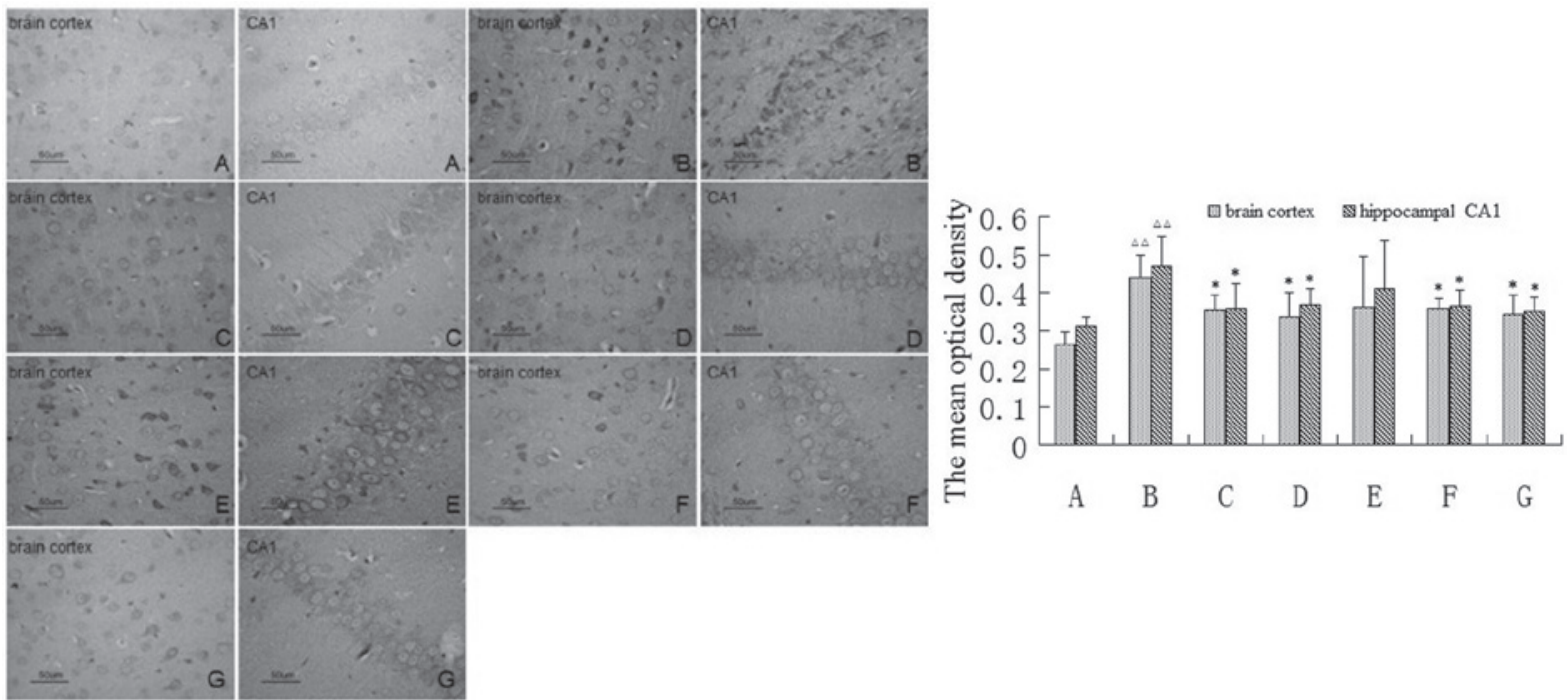

Figure 4. Effect of bilobalide (BB) on TNF- $\alpha$ immunoreactive cells in the brain cortex and the hippocampal CA1 region of vascular dementia (VD) rats (magnification, x400). (A) Sham-surgery, (B) model, (C) Nimo, (D) Hupa, (E) BB $2 \mathrm{mg} / \mathrm{kg}$, (F) BB $4 \mathrm{mg} / \mathrm{kg}$ and (G) BB $8 \mathrm{mg} / \mathrm{kg}$ groups (n=5 rats/group).

$\triangle \triangle \mathrm{P}<0.01$ vs. sham-surgery group; ${ }^{* *} \mathrm{P}<0.05$ vs. model group. TNF- $\alpha$, tumor necrosis factor- $\alpha$.

$(\mathrm{P}<0.01)$. BB (4 and $8 \mathrm{mg} / \mathrm{kg}$ ), Nimo and Hupa treatment obviously decreased the expression of TNF- $\alpha$ in these regions of $\mathrm{VD}$ rats $(\mathrm{P}<0.05)$ (Fig. 4).

\section{Discussion}

The 2-VO rat is a suitable model for investigating the 
pathophysiology of learning and memory impairment in human dementia, as well as for assessing the therapeutic potential and/or exploring possible mechanisms of putative anti-dementia drugs (13). BB, a sesquiterpene trilactone constituent of Ginkgo biloba leaf extracts, has been suggested to exert protective and trophic effects on neurons $(8,9,14)$. MWM has been proven to be an effective method for the ethological evaluation of rats, and has been used to evaluate the spatial learning and memory abilities of rats (15). In the present study, the spatial learning and memory abilities of rats were decreased in the model group, while they were found to be improved following BB $(4$ and $8 \mathrm{mg} / \mathrm{kg})$ treatment. These results suggest that daily administration of $\mathrm{BB}$ rescues cognitive deficits in VD rats.

The mechanism underlying the permanent occlusion of both common carotid arteries responsible for inducing learning and memory deficits is intricate. Neuronal apoptosis has been found to be related to deficits in spatial learning and memory in rats induced by chronic cerebral hypoperfusion (16). Hippocampus plays an important role in learning and memory processing, and is highly sensitive to ischemic insults $(11,17)$. Cerebral ischemia inducing neuronal degeneration has also been observed in other structures, such as striatum, brain cortex and thalamus $(18,19)$. In the present study, histological changes and neuronal apoptosis in the brain cortex and the hippocampal CA1 region of rats were investigated. Histological examination plays an important role in the evaluation of neuronal damage and drug action (20). H\&E and Hoechst 33258 staining showed that the number of apoptotic cells was significantly increased in the rats of the model group, and that $\mathrm{BB}(4$ and $8 \mathrm{mg} / \mathrm{kg})$ treatment alleviated the neuronal impairment and apoptosis. These results suggest that $\mathrm{BB}$ has an antiapoptotic activity.

Oxidative stress is involved in neuronal apoptosis (21). It is well known that free radicals participate in the regulation of neuron degeneration and apoptosis, and are potentially involved in the pathogenesis of neurodegenerative diseases such as VD $(22,23)$. MDA is an important biomarker of lipid peroxidation that is induced by reactive oxygen species. Specific quantification of MDA is able to determine the extent of lipid damage as a result of oxidative stress in a variety of lipid systems, such as plasma, organs and cell membranes (24). Free radicals cause oxidative damage to important biological molecules. Consequently, organisms exhibit increased SOD, catalase and glutathione peroxidase activities in response to oxidative stress (25). Increased SOD activity could be regarded as an indication that the antioxidant mechanism of the brain is activated in response to oxidative stress (26). Free radicals directly damage antioxidant enzymes and reduce their activities (27). In the present study, GSH content was found to be significantly decreased in VD rats. Therefore, the increment in MDA content and SOD activity found in the rats of the model group suggests an obviously increased oxidative process, while the decrease in the GSH content indicates the presence of deficiencies in the endogenous antioxidant ability. Nitric oxide (NO) might exacerbate the damage by enhancing the post-ischemic release of excitatory neurotransmitters (28). Changes in the level of NO produced by NOS occur in acute cerebral ischemia and Alzheimer's disease (AD) (29). In the present study, BB $(2,4$ and $8 \mathrm{mg} / \mathrm{kg})$ significantly increased
SOD activity and GSH content, and decreased MDA content and NOS activity in VD rats. Previous studies have demonstrated that agents with antioxidant and radical scavenger properties have been effective in the treatment of VD in animals or in clinical trials (30-32). In the present study, learning and memory dysfunction is suggested to have been alleviated by BB due to its antioxidant properties, since oxidative stress in all cases plays an important role in 2-VO-induced neurodegenerative processes (33).

TNF- $\alpha$, a pro-inflammatory cytokine that is produced by macrophages, adipocytes and astrocytes, appears to be an unspecific but potent factor in the development of several psychiatric diseases, including depression and dementia. Elevated TNF- $\alpha$ levels have been found in the cerebrospinal fluid of patients with $\mathrm{AD}$ and VD, suggesting an important role of TNF- $\alpha$ production by the central nervous system in dementia (34). TNF- $\alpha$ has been shown to induce neuronal death in several neurodegenerative disorders including $A D$ and VD (35). The results of the present study have shown that BB (4 and $8 \mathrm{mg} / \mathrm{kg}$ ) significantly decreased the expression of TNF- $\alpha$ in the brain cortex and the hippocampal CA1 region of VD rats. This suggests that the inhibitory effect of $\mathrm{BB}$ on neuronal apoptosis is associated with the regulation of TNF- $\alpha$ expression by BB.

In conclusion, the present study has demonstrated that BB has a potential antioxidant activity, inhibits neuronal apoptosis and reduces the expression of TNF- $\alpha$ in the brain cortex and the hippocampal CA1 region of VD rats. These effects might be related to its antioxidant and antiapoptotic activities. However, additional studies are needed for the elucidation of the detailed mechanism between the two activities of BB.

\section{Acknowledgements}

This study was supported by the Doctor Foundation of Anhui Medical University (XJ201011) and the Teaching Quality Project of Anhui Higher Education (20101941). The authors thank Dake Huang, Shan Huang and Li Gui (Synthetic Laboratory of Basic Medicine College, Anhui Medical University, Hefei, China) for their excellent technical assistance.

\section{References}

1. Román GC: Vascular dementia: distinguishing characteristics, treatment, and prevention. J Am Geriatr Soc 51: S296-S304, 2003.

2. Ahlemeyer B and Krieglstein J: Neuroprotective effects of Ginkgo biloba extract. Cell Mol Life Sci 60: 1779-1792, 2003.

3. Chandrasekaran K, Mehrabian Z, Spinnewyn B, Chinopoulos C, Drieu K and Fiskum G: Neuroprotective effects of bilobalide, a component of Ginkgo biloba extract (EGb 761) in global brain ischemia and in excitotoxicity-induced neuronal death. Pharmacopsychiatry 36 (Suppl 1): S89-S94, 2003.

4. Tanaka Y, Marumo T, Omura T and Yoshida S: Serum S100B indicates successful combination treatment with recombinant tissue plasminogen activator and MK-801 in a rat model of embolic stroke. Brain Res 1154: 194-199, 2007.

5. Smith JV and Luo Y: Studies on molecular mechanisms of Ginkgo biloba extract. Appl Microbiol Biotechnol 64: 465-472, 2004.

6. Klein J, Chatterjee SS and Löffelholz K: Phospholipid breakdown and choline release under hypoxic conditions: inhibition by bilobalide, a constituent of Ginkgo biloba. Brain Res 755: 347-350, 1997. 
7. Weichel O, Hilgert M, Chatterjee SS, Lehr M and Klein J: Bilobalide, a constituent of Ginkgo biloba, inhibits NMDA-induced phospholipase A2 activation and phospholipid breakdown in rat hippocampus. Naunyn Schmiedebergs Arch Pharmacol 360: 609-615, 1999.

8. Rossi R, Basilico F, Rossoni G, Riva A, Morazzoni P and Mauri PL: Liquid chromatography/atmospheric pressure chemical ionization ion trap mass spectrometry of bilobalide in plasma and brain of rats after oral administration of its phospholipidic complex. J Pharm Biomed Anal 50: 224-227, 2009.

9. Defeudis FV: Bilobalide and neuroprotection. Pharmacol Res 46: 565-568, 2002

10. Sarti C, Pantoni L, Bartolini L and Inzitari D: Persistent impairment of gait performances and working memory after bilateral common carotid artery occlusion in the adult Wistar rat. Behav Brain Res 136: 13-20, 2002.

11. Ni J, Ohta H, Matsumoto K and Watanabe H: Progressive cognitive impairment following chronic cerebral hypoperfusion induced by permanent occlusion of bilateral carotid arteries in rats. Brain Res 653: 231-236, 1994.

12. Janus C, Pearson J, McLaurin J, et al: A beta peptide immunization reduces behavioural impairment and plaques in a mode of Alzheimer's disease. Nature 408: 979-982, 2000.

13. Sarti C, Pantoni L, Bartolini L and Inzitari D: Cognitive impairment and chronic cerebral hypoperfusion: what can be learned from experimental models. J Neurol Sci 203-204: 263-266, 2002

14. Bruno C, Cuppini R, Sartini S, Cecchini T, Ambrogini P and BombardelliE: Regeneration of motor nerves in bilobalide-treated rats. Planta Med 59: 302-307, 1993.

15. D'Hooge R and De Deyn PP: Applications of the Morris water maze in the study of learning and memory. Brain Res Brain Res Rev 36: 60-90, 2001.

16. Bennett SA, Tenniswood M, Chen JH, Davidson CM, Keyes MT, Fortin T and Pappas BA: Chronic cerebral hypoperfusion elicits neuronal apoptosis and behavioral impairment. Neuroreport 9: 161-166, 1998

17. Pappas BA, de la Torre JC, Davidson CM, Keyes MT and Fortin T: Chronic reduction of cerebral blood flow in the adult rat: late-emerging CA1 cell loss and memory dysfunction. Brain Res 708: 50-58, 1996.

18. Freund TF, Buzsáki G, Leon A, Baimbridge KG and Somogyi P: Relationship of neuronal vulnerability and calcium binding protein immunoreactivity in ischemia. Exp Brain Res 83: 55-66, 1990.

19. Pereira LO, Nabinger PM, Strapasson AC, Nardin P, Gonçalves CA, Siqueira IR and Netto CA: Long-term effects of environmental stimulation following hypoxia-ischemia on the oxidative state and BDNF levels in rat hippocampus and frontal cortex. Brain Res 1247: 188-195, 2009.

20. Hunter AJ, Mackay KB and Rogers DC: To what extent have functional studies of ischaemia in animals been useful in the assessment of potential neuroprotective agents? Trends Pharmacol Sci 19: 59-66, 1998.
21. Coyle JT and Puttfarcken P: Oxidative stress, glutamate, and neurodegenerative disorders. Science 262: 689-695, 1993.

22. Markesbery WR: Oxidative stress hypothesis in Alzheimer's disease. Free Radic Biol Med 23: 134-147, 1997.

23. Chong ZZ, Li F and Maiese K: Oxidative stress in the brain novel cellular targets that govern survival during neurodegenerative disease. Prog Neurobiol 75: 207-246, 2005.

24. Lazzarino G, Tavazzi B, Di Pierro D, Vagnozzi R, Penco M and Giardina B: The relevance of malondialdehyde as a biochemical index of lipid peroxidation of postischemic tissues in the rat and human beings. Biol Trace Elem Res 47: 165-170, 1995.

25. Halliwell B: Reactive oxygen species in living systems: source, biochemistry, and role in human disease. Am J Med 91: S14-S22, 1991.

26. Bannister JV, Bannister WH and Rotilio G: Aspects of the structure, function, and applications of superoxide dismutase. CRC Crit Rev Biochem 22: 111-180, 1987.

27. Escobar JA, Rubio MA and Lissi EA: Sod and catalase inactivation by singlet oxygen and peroxyl radicals. Free Radic Biol Med 20: 285-290, 1996

28. Montague PR, Gancayco CD, Winn MJ, Marchase RB and Friedlander MJ: Role of NO production in NMDA receptor-mediated neurotransmitter release in cerebral cortex. Science 263: 973-977, 1994.

29. Iadecola C: Bright and dark sides of nitric oxide in ischemic brain injury. Trends Neurosci 20: 132-139, 1997.

30. Tabet N, Mantle D, Walker Z and Orrell M: Vitamins, trace elements, and antioxidant status in dementia disorders. Int Psychogeriatr 13: 265-275, 2001

31. DeFeudis FV and Drieu K: Ginkgo biloba extract (EGb 761) and CNS functions: basic studies and clinical applications. Curr Drug Targets 1: 25-58, 2000.

32. Xiong Z, Liu C, Wang F, Li C, Wang W, Wang J and Chen J: Protective effects of breviscapine on ischemic vascular dementia in rats. Biol Pharm Bull 29: 1880-1885, 2006.

33. Farkas E, Luiten PG and Bari F: Permanent, bilateral common carotid artery occlusion in the rat: a model for chronic cerebral hypoperfusion-related neurodegenerative diseases. Brain Res Rev 54: 162-180, 2007.

34. Tarkowski E, Blennow K, Wallin A and Tarkowski A: Intracerebral production of tumor necrosis factor-alpha, a local neuroprotective agent, in Alzheimer disease and vascular dementia. J Clin Immunol 19: 223-230, 1999.

35. Zuliani G, Ranzini M, Guerra G, Rossi L, Munari MR, Zurlo A, Volpato S, Atti AR, Blè A and Fellin R: Plasma cytokines profile in older subjects with late onset Alzheimer's disease or vascular dementia. J Psychiatr Res 41: 686-693, 2007. 